\title{
On the Synthesis of Feasible and Prehensile Robotic Grasps
}

\author{
Carlos Rosales, Raúl Suárez, Marco Gabiccini, Antonio Bicchi
}

\begin{abstract}
This work proposes a solution to the grasp synthesis problem, which consist of finding the best hand configuration to grasp a given object for a specific manipulation task while satisfying all the necessary constraints. This problem had been divided into sequential sub-problems, including contact region determination, hand inverse kinematics and force distribution, with the particular constraints of each step tackled independently. This may lead to unnecessary effort, such as when one of the problems has no solution given the output of the previous step as input. To overcome this issue, we present a kinestatic formulation of the grasp synthesis problem that introduces compliance both at the joints and the contacts. This provides a proper framework to synthesize a feasible and prehensile grasp by considering simultaneously the necessary grasping constraints, including contact reachability, object restraint, and force controllability. As a consequence, a solution of the proposed model results in a set of hand configurations that allows to execute the grasp using only a position controller. The approach is illustrated with experiments on a simple planar hand using two fingers and an anthropomorphic robotic hand using three fingers.
\end{abstract}

keywords: grasp synthesis, robotic hands, stiffness method.

\section{INTRODUCTION}

Mechanical anthropomorphic hands have been introduced for the last decades with the idea of performing dexterous manipulation tasks, that is, moving an object within the hand by means of finger motions [1]-[3]. Fig. 1] shows two different designs of a robotic hand with four and five fingers. In order to achieve this complex coordination autonomously, the hand must be previously commanded to grasp the object such that the subsequent manipulation can occur. Whether the task defines how the object should be grasped, or it is the grasping configuration that allow certain types of tasks is always a matter of fruitful discussions [4]. In both cases, the initial grasp configuration, the very first step in dexterous manipulation, is a crucial step to accomplish a given task. Finding such initial grasp configuration is called the grasp synthesis problem [1]. Although in most of the works dealing with dexterous manipulation using robotic hands it is assumed to be given [3, 5]-[9], a systematic and

C. Rosales and R. Suárez are with the Institut d'Organizació i Control de Sistemes Industrials, UPC, Barcelona, Spain, \{carlos.rosales-gallegos, raul.suarez\}@upc.edu.

C. Rosales is also with the Institut de Robòtica i Informàtica Industrial, CSIC-UPC, Barcelona, Spain. M. Gabiccini and A Bicchi are with the Interdept. Research Center "E. Piaggio", University of Pisa, Pisa, Italy, m.gabiccini@ing.unipi.it, bicchidcentropiaggio.unipi.it. A. Bicchi is also with the Istituto Italiano di Tecnologia, Genova, Italy.

This work was partially supported by the CICYT projects DPI2010-18449, DPI2010-15446 and DPI2011-22471, and by the European Commission under CP grants no. 248587, "THE Hand Embodied", and no. 270350, "ROBLOG", within the FP7-ICT-2009-4-2-1 program "Cognitive Systems and Robotics".
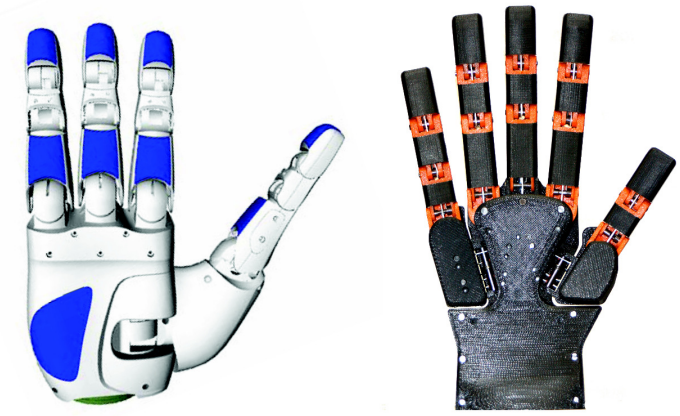

Fig. 1. Different robotic hand designs, the Schunk Anthropomorphic hand, based on the DLR II hand, with four fingers (left), and THE first hand developed at the Centro E. Piaggio with five fingers (right).

robust way to its definition is a wide open issue [4]. Solution to sub-problems exist forming a sequential approach. First, one seeks for the contact locations that restrain the object motions [10]-[12]. Second, one tries to find the hand configuration that reach to those locations [13]-[16], being the solution of the first problem used as input. Third, one selects the internal grasping forces given a change in the external force applied on the object, which assumes both that the first two problems were solved and the configuration is already in static equilibrium [17]-[21]. Recent research works have already started to merge some of the mentioned problems [22]-[25], however, they either miss some of the necessary constraints, limit the approach to planar grasps, or use a reduced number of fingers. A similar problematic is found as well on the synthesis of stable configurations of tensegrities [26].

In this work, we propose a solution to the grasp synthesis problem using a kinestatic formulation, inspired by the notion of soft synergies introduced in [21] and [27]. The approach effectively introduces an elastic model of the hand, whereby the physical hand is attracted toward a reference hand through a set of virtual springs at the joints (representing the compliance of the musculo-skeletal system), while being repelled by the object through contacts, which are also supposed to be compliant. This model allows to tackle simultaneously the necessary constraints when synthesizing a feasible and prehensile grasp. These include the contact reachability, i.e. the hand must accommodate to touch the object surface at specified contact points, the object restraint, i.e. the object motion due to external perturbations is prevented by applying valid contact forces according to the contact model, and force controllability constraint, i.e. the valid contact forces must be compensated by joint torques 
and not entirely by the structure. As a consequence, the solution results in a set of hand configurations that allow to execute the grasp using only a position controller, since the problem variables are configuration values. Since the model is compatible with the notion of soft synergies, it allows for the reference hand to be controlled in a simplified way through coupling joint motions, according e.g. to empirical data on hand posture correlations or synergies [27]-[29].

The paper is organized as follows: Section $\amalg$ presents the formulation of the feasible and prehensile grasp synthesis problem. Section III casts the formulation as an optimization problem using a potential energy-based criterion. Section IV shows the experimental results that validate the approach. Finally, Section $\nabla$ wraps up the conclusions and remarks points deserving further study.

\section{Kinestatic Formulation OF THE GRASP SyNTHESIS PROBLEM}

The grasp synthesis problem consist of finding a feasible and prehensile grasp configuration. The formulation involves the specification of three hand configurations, as shown in Fig. 2. The outer hand configuration (black) accounts for the feasibility, i.e. whether the fingertips can actually reach the object surface given the kinematic structure of the hand. The inner hand configuration (blue) the configuration given to produce joint torques that squeeze and restrain the object. The interaction yield reaction forces. In between, the middle hand configuration (green) is the actual grasping configuration where the joint torques and contact forces are balanced accounting for prehensility and the static equilibrium is achieved, whence the term kinestatic: kinematics + statics.

\section{A. Model Description and Nomenclature}

A grasp is a configuration of a hand and an object adjoined at certain contact points. For simplicity, in this paper we assume precision grasps, i.e. that only one contact per finger at the fingertip is used. Precision grasps are most often used for dextrous manipulation [30]. While the soft synergy model can easily account for inner-hand contacts and power grasps, the algorithmic complexity of our method grows with the number of contacts to place and with the number of kinematic constraints.

A robotic hand is usually composed of several articulated fingers attached to a palm. The hand palm is positioned and oriented with respect to the world using the matrix $\mathbf{T}_{H} \in S E(3)$. The hand is composed of $n$ fingers, each of them articulated through $m_{i}$ revolute joints, for $i=1, \ldots, n$, which sum up to $m=\sum_{i=1}^{n} m_{i}$ hand joints. The rotation angle of the $j$-th joint at the $i$-th finger is the joint value $q_{i j} \in \mathbb{S}$, where $\mathbb{S}$ denotes the angular nature of values. The phalanges are positioned and oriented with respect to the world using the homogeneous matrix $\mathbf{T}_{i j}$, which depends on the joint values, $q_{i j}$, for $j=1, \ldots, m_{i}$ and $i=1, \ldots, n$. Thus, by collecting all joint values in the vector $\mathbf{q}=\left(q_{11}, \ldots q_{i j} \ldots, q_{n m_{n}}\right)$, a configuration of the hand is represented by the pair $\left(\mathbf{q}, \mathbf{T}_{H}\right) \in \mathbb{S}^{m} \times S E(3)$.

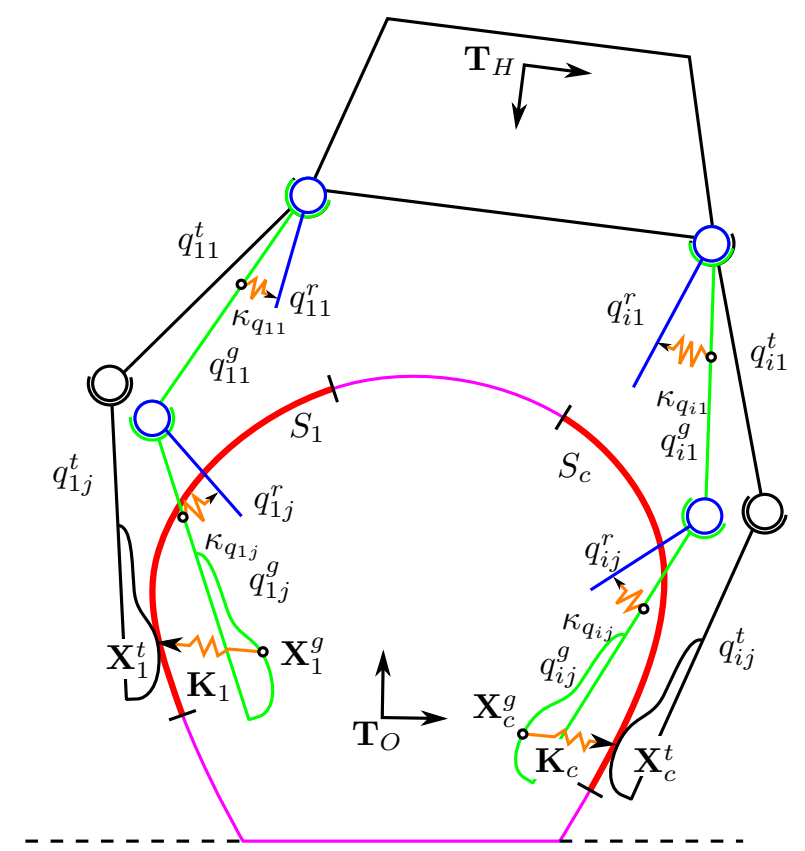

Fig. 2. Kinestatic model using springs at the joints and at the contact. The grasp is characterized by three hand configurations accounting for the feasibility and prehensility of a grasp.

There are $n$ given contact points, one on each fingertip. A reference frame, $\mathbf{X}_{c} \in S E(3)$, is placed at the contact point $\mathbf{x}_{c} \in \mathbb{R}^{3}$, for $c=1, \ldots, n$. The outward normal vector at the contact point is denoted as $\hat{\mathbf{n}}_{c}$. Both the contact point and the contact normal depend on the hand configuration $\left(\mathbf{q}, \mathbf{T}_{H}\right)$. The number of contact locations, $n$, is assumed to be, at least, the minimum required to restrain the object according to the chosen contact model (e.g. $n=7$ for non-frictional contacts spatial grasps [1]).

The object is positioned and oriented with respect to the world using the matrix $\mathbf{T}_{O} \in S E(3)$. Without loss of generality, it is fixed and coincident with the world reference frame. On its surface, there is a given contact region, $S_{c}$, for each contact point, $\mathbf{x}_{c}$, on the hand. The coordinates of a point on the region $S_{c}$ is obtained using the parametrization $\mathbf{s}_{c}\left(\gamma_{c}\right)$, where $\gamma_{c} \in \mathbb{R}^{a_{c}}$, with $a_{c}=0,1,2$, the vector of parameters defining a point, a curve or a surface, respectively. The parametric inward normal vector at the point $\mathbf{s}_{c}$, is denoted as $\hat{\mathbf{m}}_{c}\left(\boldsymbol{\gamma}_{c}\right)$.

The contact forces and joint torques are modeled using a spatial spring at the contacts and a torsional spring at the joints with known stiffness constants $\mathbf{K}_{c}$ and $\kappa_{q_{i j}}$, respectively. The rest position for the contact springs is defined by the touching configuration of the hand, $\left(\mathbf{q}^{t}, \mathbf{T}_{H}\right)$, that makes the desired contact point, $\mathbf{x}_{c}^{t}$, on each finger, reach the corresponding region, $S_{c}$, on the object. A reference configuration of the hand, $\left(\mathbf{q}^{r}, \mathbf{T}_{H}\right)$, pulls the fingers inside the object surface loading the springs at the joints. The grasping configuration, $\left(\mathbf{q}^{g}, \mathbf{T}_{H}\right)$, floats between those two, and modifies the rest position for the joint springs, while the contact frame is moved to $\mathbf{X}_{c}^{g}$, loading the contact springs and pushing the fingers out of the object back to $\mathbf{X}_{c}^{t}$. 
TABLE I

BASIC NOTATION FOR THE KINESTATIC MODEL OF A GRASP.

\begin{tabular}{ll}
\hline Sym. & Definition \\
\hline $\mathbf{T}_{H}$ & Hand reference frame \\
$\mathbf{T}_{O}$ & Object reference frame \\
$m$ & Number of joints \\
$n$ & Number of fingers and contacts \\
$q_{i j}$ & Value of the $j$-th joint at the $i$-th finger \\
$\kappa_{q_{i j}}$ & Stiffness of torsional spring of the $j$-th joint at the $i$-th finger \\
$\mathbf{q}^{t}$ & Touching joint configuration \\
$\mathbf{q}^{g}$ & Grasping joint configuration \\
$\mathbf{q}^{r}$ & Reference joint configuration \\
$\delta \mathbf{q}$ & Joint displacement from $\mathbf{q}^{g}$ to $\mathbf{q}^{r}$ \\
$\mathbf{X}_{c}^{g}$ & Contact reference frame, with origin at the point $\mathbf{x}_{c}^{g}$ \\
$\mathbf{X}_{c}^{t}$ & Contact reference frame, with origin at the point $\mathbf{x}_{c}^{t}$ \\
$\delta \mathbf{X}_{c}$ & The rigid body motion from $\mathbf{X}_{c}^{t}$ to $\mathbf{X}_{c}^{g}$ \\
$\mathbf{K}_{c}$ & Stiffness of the spatial spring at the $c$-th contact point \\
$h_{c}$ & Number of linear springs used at the $c$-th contact point \\
$S_{c}$ & Contact region on the object corresponding to the $c$-th contact point \\
$\gamma_{c}$ & Parameter that defines a point on $S_{c}$ \\
$a_{c}$ & Dimensionality of region $S_{c}$ (point, curve, surface) \\
$b_{c}$ & Orientation freedom at the $c$-th contact (normal alignment or not) \\
$\mathbf{S}_{c}$ & Contact point on the region $S_{c}$ defined by $\gamma_{c}$ \\
$\hat{\mathbf{m}}_{c}$ & Normal vector at the point $\mathbf{s}_{c}$ defined by $\gamma_{c}$ \\
$\mathbf{J}$ & Hand Jacobian evaluated at the grasping configuration \\
$\mathbf{G}$ & Grasp matrix evaluated at the grasping configuration \\
$\mathbf{K}_{q}$ & Hand stiffness matrix \\
$\mathbf{K}$ & Contact stiffness matrix \\
\hline &
\end{tabular}

\section{B. Characterizing the Feasibility}

A grasp is feasible when the touching configuration of the hand, $\left(\mathbf{q}^{t}, \mathbf{T}_{H}\right)$ makes the points on the fingertips contact properly at the corresponding regions on the object, i.e. $\mathbf{x}_{c}^{t} \in S_{c}$. Thus, the contact reachability is written as

$$
\left\|\mathbf{x}_{c}^{t}-\mathbf{s}_{c}\left(\gamma_{c}\right)\right\|_{2}=0
$$

In order to avoid interpenetrations of the fingertips on the object, the normal vectors at the contacting points are aligned by requiring

$$
\hat{\mathbf{n}}_{c}^{t} \cdot \hat{\mathbf{m}}_{c}\left(\gamma_{c}\right)=1
$$

Additionally, the position vector of the matrices $\mathbf{T}_{i j}, \mathbf{r}_{i j}$, are forced to be outside the object by requiring

$$
\hat{\mathbf{m}}_{c}^{\top}\left(\mathbf{s}_{c}-\mathbf{r}_{i j}\right)>0,
$$

which means that the projection of vector going from $\mathbf{r}_{i j}$ to $\mathbf{s}_{c}$ onto the normal at the contact point is positive.

Finally, the joint values of real robotic hands are subject to mechanical limitations. Hence, the touching configuration must fulfill the preceding constraints while the joint values stay within the valid range, that is,

$$
\mathbf{q}^{l} \leq \mathbf{q}^{t} \leq \mathbf{q}^{u}
$$

with $\mathbf{q}^{l}$ and $\mathbf{q}^{u}$ the vectors of minimum and maximum values that they can reach, respectively.

\section{Characterizing the Prehensility}

The prehensility condition is met when the object motion due to external perturbations is prevented by applying valid contact forces according to the contact model and the grasping configuration, also known as the object restrainment, and then, those contact forces are balanced by applying joint torques according to the reference configuration, also known as force controllability. In the literature, they are also known as object equilibrium and hand equilibrium constraints, respectively [25]. It is worth noting that, the prehensility, together with the assumption that $n$ is the minimum number of contacts required to restraint the object according to the contact model, yields a force-closure grasp as defined by [31].

Object Restrainment: Each contact force is modeled using a spatial springs conformed of $h_{c}$ linear springs connecting the contact frames $\mathbf{X}_{c}^{t}$ and $\mathbf{X}_{c}^{g}$ (see Fig. 3). Thus, we express the effect of these springs acting on the object, i.e. the $c$-th contact force, as the sum of all spring forces as $\mathbf{w}_{c}=\left[\begin{array}{lll}\hat{\mathbf{p}}_{1, c} & \cdots & \hat{\mathbf{p}}_{h_{c}, c}\end{array}\right] \boldsymbol{\lambda}_{c}$, where $\hat{\mathbf{p}}_{k, c} \in \mathbb{R}^{6}$ is the supporting line of the $k$-th spring passing through the contact point $\mathbf{x}_{c}^{g}$, and $\boldsymbol{\lambda}_{c}=\left[\begin{array}{lll}\lambda_{1, c} & \ldots & \lambda_{h_{c}, c}\end{array}\right]^{\top}$ collects the force magnitudes of the springs obtained as $\lambda_{k, c}=-\kappa_{k, c} d_{k, c}$, where $d_{k, c}$ is the spring elongation and $\kappa_{k, c}$ the stiffness constant of the $k$-th spring. Thus, the magnitude of the contact force can be written as $\boldsymbol{\lambda}_{c}=-\tilde{\mathbf{K}}_{c} \mathbf{d}_{c}$ using the diagonal matrix $\tilde{\mathbf{K}}_{c}=\operatorname{diag}\left(\kappa_{1}, \ldots, \kappa_{h_{c}}\right)$ and $\mathbf{d}_{c}=\left[\begin{array}{lll}d_{1, c} & \ldots & d_{h_{c}, c}\end{array}\right]^{\top}$. Then, introducing the matrix $\mathbf{P}_{c}=\left[\begin{array}{lll}\hat{\mathbf{p}}_{1, c} & \ldots & \hat{\mathbf{p}}_{h_{c}, c}\end{array}\right]$, we express the $c$-th contact force as

$$
\mathbf{w}_{c}=-\mathbf{P}_{c} \tilde{\mathbf{K}}_{c} \mathbf{d}_{c}
$$

The displacement that goes from $\mathbf{X}_{c}^{t}$ to $\mathbf{X}_{c}^{g}$ can be parametrized using six independent variables, known as the exponential coordinates [3], if it satisfies

$$
\mathbf{X}_{c}^{g}=e^{\left(\delta \mathbf{X}_{c}\right)} \mathbf{X}_{c}^{t}
$$

where $e^{\left(\delta \mathbf{X}_{c}\right)}$ is the exponential map representing the relative finite rigid body displacement between them, $\delta \mathbf{X}_{c} \in \operatorname{se}(3)$. The spring elongations is obtained by projecting the displacement onto the supporting lines of the springs as

$$
\mathbf{d}_{c}=\mathbf{P}_{c}^{\top} \delta \mathbf{X}_{c} \text {. }
$$

Substituting (7) in (5) yields the expression of the contact force as a function of the touching and grasping configuration,

$$
\mathbf{w}_{c}=-\mathbf{K}_{c} \delta \mathbf{X}_{c},
$$

where $\mathbf{K}_{c}=\mathbf{P}_{c} \tilde{\mathbf{K}}_{c} \mathbf{P}_{c}^{\top}$.

Since we are assuming that $n$ is the minimum number of contact points required, we just need to enforce the equilibrium of all contact forces, i.e. $\sum_{c=1}^{n} \mathbf{w}_{c}=0$. Thus, building the matrix $\mathbf{G}=\left[\begin{array}{lll}\mathbf{P}_{1} & \ldots & \mathbf{P}_{n}\end{array}\right]$, the block diagonal matrix $\mathbf{K}=\operatorname{blkdiag}\left(\tilde{\mathbf{K}}_{1}, \ldots, \tilde{\mathbf{K}}_{n}\right)$, the block diagonal matrix $\mathbf{P}=\operatorname{blkdiag}\left(\mathbf{P}_{1}, \ldots, \mathbf{P}_{n}\right)$, and collecting all contact displacements in $\delta \mathbf{X}=\left[\begin{array}{lll}\delta \mathbf{X}_{1}^{\top} & \ldots & \delta \mathbf{X}_{n}^{\top}\end{array}\right]^{\top}$, the object restrainment can be expressed as

$$
\mathbf{G K P}^{\top} \delta \mathbf{X}=\mathbf{0} .
$$

Additionally, the contact forces must comply with the contact model. Typical contact models in grasping include the point contact without friction (PC), point contact with 


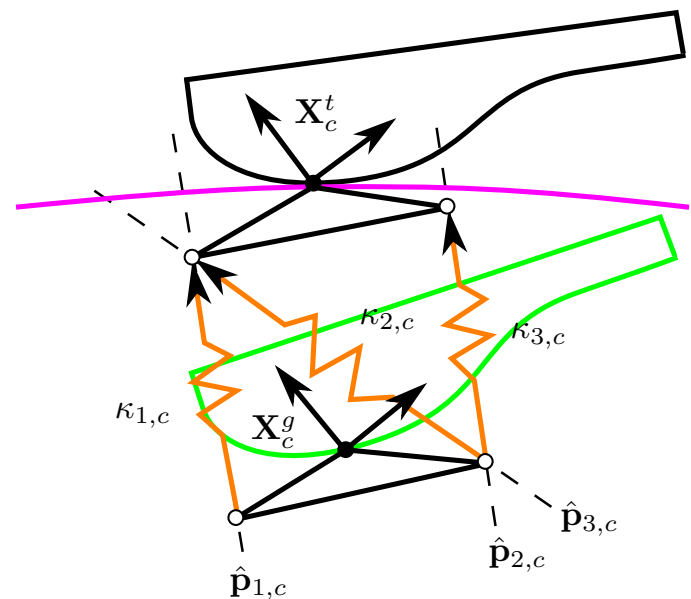

Fig. 3. Representation of the spatial spring placed at the contact locations In this planar case, the contact model is SF, hence there are $h_{c}=3$ linear springs. The triangles are drawn for clarity, however, the vertices coincident with the contact point, making $\mathbf{p}_{1, c}, \mathbf{p}_{2, c}$ and $\mathbf{p}_{3, c}$, pass through the origin of $\mathbf{X}_{c}$. The rest position of the springs correspond to the configuration where $\mathbf{X}_{c}^{g}=\mathbf{X}_{c}^{t}$.

friction (PCWF), and contact with a soft finger (SF) [1]. They can be implemented by considering springs only at the constrained directions, with $h_{c}=1,3,4$, depending whether we use a PC, PCWF, or SF, respectively. Without loss of generality, the supporting lines of the springs are chosen such that $\hat{\mathbf{p}}_{1, c}$ indicates a translation along the inward normal direction, $\mathbf{p}_{2, c}$ and $\hat{\mathbf{p}}_{3, c}$ indicates translations along the tangent directions, and $\hat{\mathbf{p}}_{4, c}$ indicates a rotation about the normal direction. Thus, the linear and torsional friction coefficients, $\mu_{c}$ and $\nu_{c}$, define an additional constraint on the vector $\mathbf{w}_{c}=\left[\begin{array}{lll}w_{1, c} & \ldots & w_{6, c}\end{array}\right]$, which must belong to the generalized friction cone

$$
\mathcal{C}_{c}=\left\{\mathbf{w}_{c} \in \mathbb{R}^{6} \mid\left\|\mathbf{w}_{c}\right\|_{\Delta} \leq w_{1, c}\right\},
$$

where $\left\|\mathbf{w}_{c}\right\|_{\Delta}$ can take the form $0, \frac{1}{\mu_{c}} \sqrt{w_{2, c}^{2}+w_{3, c}^{2}}$ or $\frac{1}{\mu_{c}} \sqrt{w_{2, c}^{2}+w_{3, c}^{2}}+\frac{1}{\nu_{c}}\left|w_{4, c}\right|$ depending on whether we use the PC, PCWF, or SF model, respectively, as proposed in [1].

Force Controllability: Each joint torque is modeled using a torsional spring connecting the grasping and the reference configuration at the joints. The resultant force due to the spring elongation is written as

$$
\mathbf{w}_{i j}=\hat{\mathbf{z}}_{i j} \tau_{i j},
$$

where $\hat{\mathbf{z}}_{i j}$ is the supporting line that coincides with the joint rotation axis at the grasping configuration, and $\tau_{i j}$ is the torque magnitude obtained as $\tau_{i j}=\kappa_{i j} d_{i j}$, where $d_{i j}$ is the spring elongation. The joint displacement of the $i$-th finger is expressed as $\delta \mathbf{q}_{i}=\mathbf{q}_{i}^{r}-\mathbf{q}_{i}^{g}$, where the subscript $i$ indicates that only the $m_{i}$ joints of the $i$-th finger are used. Thus, introducing the matrix $\mathbf{Z}_{i}=\left[\begin{array}{lll}\hat{\mathbf{z}}_{i, 1}^{\top} & \ldots & \hat{\mathbf{z}}_{i, m_{i}}^{\top}\end{array}\right]^{\top}$, the joint torque magnitudes that result of applying a force $\mathbf{w}_{c}$ at the $c$ th contact point is

$$
\mathbf{K}_{q_{i}} \delta \mathbf{q}_{\mathbf{i}}=\mathbf{Z}_{i}^{\top} \mathbf{w}_{c},
$$

where $\mathbf{K}_{q_{i}}=\operatorname{diag}\left(\kappa_{q_{i 1}}, \ldots, \kappa_{q_{i m_{i}}}\right)$, for $c=1, \ldots, n$ and $i=c$ in turns.

Finally, we must ensure that the contact forces are compensated by joint torques. Since the fingers are independent, the force applied at the $c$-th should be compensated by torques at the $i$-th finger, with $i=c$. Thus, introducing the block diagonal matrix $\mathbf{J}=\operatorname{blkdiag}\left(\mathbf{Z}_{1}, \ldots, \mathbf{Z}_{n}\right)$ and the vector $\mathbf{w}=\left[\begin{array}{lll}\mathbf{w}_{1}^{\top} & \ldots & \mathbf{w}_{n}^{\top}\end{array}\right]^{\top}=\mathbf{P K} \mathbf{P}^{\top} \delta \mathbf{X}$, the hand force controllability can be expressed as

$$
\mathbf{K}_{q} \delta \mathbf{q}=\mathbf{J}^{\top} \mathbf{P} \mathbf{K} \mathbf{P}^{\top} \delta \mathbf{X},
$$

where $\mathbf{K}_{q}$ and $\delta \mathbf{q}$ consider all joints ordered accordingly to the corresponding row of $\mathbf{J}^{\top}$.

In addition, the grasping configuration must be reached by the actual hand, hence the joint value limitations are again applicable here as

$$
\mathbf{q}^{l} \leq \mathbf{q}^{g} \leq \mathbf{q}^{u}
$$

Finally, the joint torques are subject to mechanical limitations as well, written as

$$
\left|\mathbf{K}_{q} \delta \mathbf{q}\right| \leq \boldsymbol{\tau}^{u},
$$

with $\tau^{u}$ the vector of maximum torque that the joint motors can exert, and the absolute value and the inequality must be read component-wise. Even when $\mathbf{q}^{r}$ is not subject to the mechanical limitations, the fingers need to be pushing against the object, enforcing a minimum torque $\tau^{l}$ by including

$$
\left|\mathbf{K}_{q} \delta \mathbf{q}\right| \geq \boldsymbol{\tau}^{l} .
$$

\section{System Overview and Dimension Analysis}

A grasp configuration $\mathbf{y}=\left(\mathbf{q}^{r}, \mathbf{q}^{g}, \mathbf{q}^{t}, \mathbf{T}_{h}, \boldsymbol{\gamma}\right)$ is feasible and prehensile if it fulfills (1), (2), (9), and (13) collected in

$$
\mathbf{M}_{\mathrm{eq}}(\mathbf{y})=\mathbf{0},
$$

and (3), (10), (15), and (16), transformed in less-than-equal inequalities and collected in

$$
\mathbf{M}_{\text {ineq }}(\mathbf{y}) \leq \mathbf{0},
$$

while staying within the valid ranges defined by (4) and (14), for $c=1, \ldots, n$ contacts, $j=1, \ldots, m_{i}$ joints and $i=c$ (in turns) fingers.

The number of variables are the internal degrees of freedom $n_{v}=3 m+\sum_{c=1}^{n} a_{c}+\sum_{c=1}^{n} b_{c}$, where $b_{c}$ depends on the orientation constraints, such as the alignment of the normal vector. The number of algebraic constraints is $n_{e}=D(n-1)+m+D$, i.e. the constraint due to the $(n-1)$ independent loops, and the $D$ and $m$ equations from the prehensility constraints, with $D$ is 3 and 6 for the planar and spatial case respectively. Assuming $a=a_{c}$ and $b=b_{c}$, for $c=1, \ldots, n$, the dimension of the solution space is then $n_{s}=n_{v}-n_{e}=2 m+(a+b-D) n$. In general, this number is greater than zero and relatively high. 


\section{CRITERION FOR UNIQUENESS AND SOLUTION STRATEGY}

There may be multiple solutions due to high nonlinearities in the constraints, even for 0-dimensional solution spaces. Thus, we propose the potential energy of the springs at the joints as the criterion to select among the possible solutions expressed as

$$
\Psi(\mathbf{y})=\frac{1}{2} \delta \mathbf{q}^{\top} \mathbf{K}_{q} \delta \mathbf{q} .
$$

It is worth noting that, when the constraints are met, the substitution of (13) in (19), yields

$$
\Psi^{\prime}(\mathbf{y})=\frac{1}{2} \delta \mathbf{q}^{\top}\left(\mathbf{J}^{\top} \mathbf{P} \mathbf{K} \mathbf{P}^{\top} \delta \mathbf{X}\right)
$$

and additionally, $\delta \mathbf{X} \approx \mathbf{J}\left(\mathbf{q}^{g}-\mathbf{q}^{t}\right)$ for small joint displacements. Thus, introducing the block diagonal matrix $\mathbf{K}^{\prime}=\mathbf{P K} \mathbf{P}^{\top}$, 20, becomes

$$
\Psi^{\prime \prime}(\mathbf{y})=\frac{1}{2}\left(\mathbf{q}^{r}-\mathbf{q}^{g}\right)^{\top} \mathbf{J}^{\top} \mathbf{K}^{\prime} \mathbf{J}\left(\mathbf{q}^{g}-\mathbf{q}^{t}\right) .
$$

By comparing (21) and (19), the criterion can be rewritten as

$$
\Psi^{\prime \prime \prime}(\mathbf{y})=\left\|\mathbf{K}_{q}-\mathbf{J}^{\top} \mathbf{K}^{\prime} \mathbf{J}\right\|,
$$

i.e. when the constraints are met and joint displacements are small, the criterion selects the configuration $\mathbf{y}$ in which the contact stiffness seen from the joints equals the joint stiffness.

The problem can be casted as: Given a hand with $n$ articulated fingers to grasp an object, with a kinematic configuration defined by the pair $\left(\mathbf{q}, \mathbf{T}_{h}\right)$, a contact on the fingertip $\mathbf{X}_{c}$, its corresponding contact region on the object surface $S_{c}, m$ joint spring stiffnesses $\kappa_{i j}$ and $n h_{c}$ contact spring stiffnesses $\kappa_{k, c}$, and friction coefficients $\mu_{c}$ and $\nu_{c}$, find a configuration $\mathbf{y}$ that minimizes the objective function (22) subject to the constraints (17, 18), (4), and (14). This non-linear optimization problem is in the form required by the MATLAB routine fmincon. We select the SQP algorithm due to its ability to work out of the solution manifold using a feasibility reformulation. This slows down the process, however it is desired when the method is starting and the configurations are far from satisfying the constraints [32].

\section{EXPERIMENTS AND RESULTS}

The method is illustrated here with two experiments. The first one consists of a simple planar hand grasping an ellipse, and the second one, of a complex robotic hand grasping an ellipsoid. The details are shown next.

\section{Example 1. A simple planar hand grasping an ellipse}

In this example, we use a simple planar hand with $n=2$ fingers, and $m_{i}=2$ joints, for a total of $m=4$ joints. The object is an ellipse, and the contact regions cover fully the ellipse boundary. The normal vectors are not aligned at the contact point, hence $b_{c}=1$. The dimension of the solution space in this case is $n_{s}=6$. The kinematic structure, spring constants and friction coefficients needed to write the grasp
TABLE II

PARAMETERS FOR EXAMPLE 1.

a) Kinematic structure and limit values

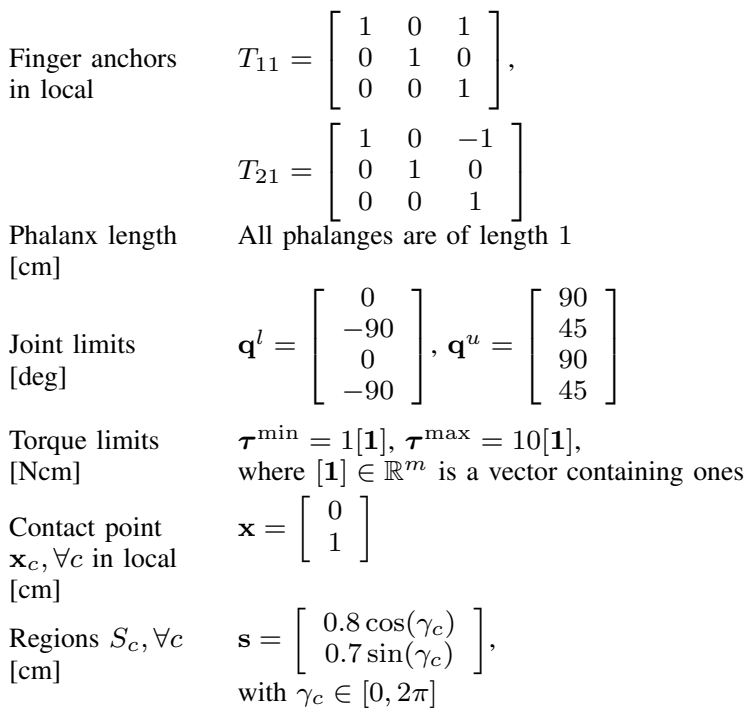

b) Coefficients

Joint stiffness $\quad \mathbf{K}_{q}=\operatorname{blkdiag}(1,1,1,1)$

[N/rad]

Friction

(PCWF) $\mu_{c}, \forall c$

Contact stiffness

$\tilde{\mathbf{K}}_{c}, \forall c[\mathrm{~N} / \mathrm{cm}]$

$\mu=0.5$

$\tilde{\mathbf{K}}=\operatorname{blkdiag}(1,1)$

synthesis problem as stated in Section III are shown in Table II In this case, the initial guesses are randomly generated, but biased towards the mean value of the limit values of the variables. The results using the proposed method from two different initial guesses are shown in Fig. 4. The relation between the touching, grasping and reference configuration can be appreciated, for instance, in the left figure, where most of the work in the right finger is done by the outer joint, not the case for the left finger, as expected from their respective touching configurations and the need of pushing against each other. For a different touching configuration and the same pushing requirement, which is the second solution found, the grasping and reference configurations are different. Note, in this case, the little work made by the joints in the left finger, where most of the contact force is absorbed by the structure, that traduces into high Cartesian stiffness, requiring less load in the joint spring. Though the hand is symmetric and their parameters are symmetric, the solutions are not symmetrical due to the randomness for their initial guesses.

\section{Example 2. An anthropomorphic hand grasping an ellipsoid}

The second example employs the Schunk anthropomorphic hand shown at the right of Fig. 1. The grasp uses $n=3$ fingers (three out of the four available), with $m_{1}=m_{2}=3$ and $m_{3}=4$ joints, for a total of $m=10$. The object is an ellipsoid, and the contact regions cover fully its surface. The normal vectors must be aligned, hence $b_{c}=1$. The 

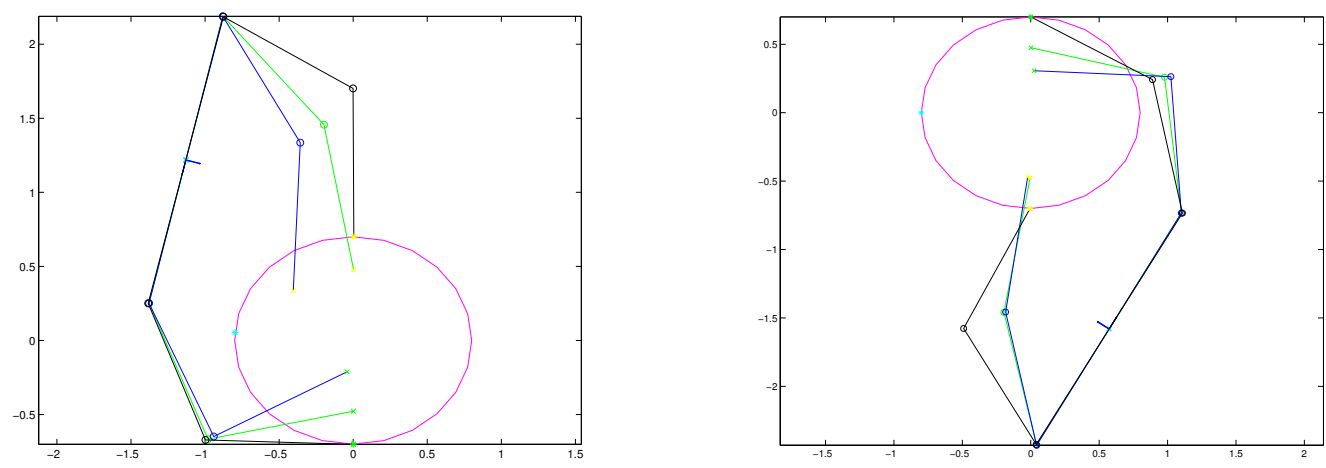

Fig. 4. Two solutions obtained for the simple hand satisfying all constraints. The color code corresponds to that of Fig. 2

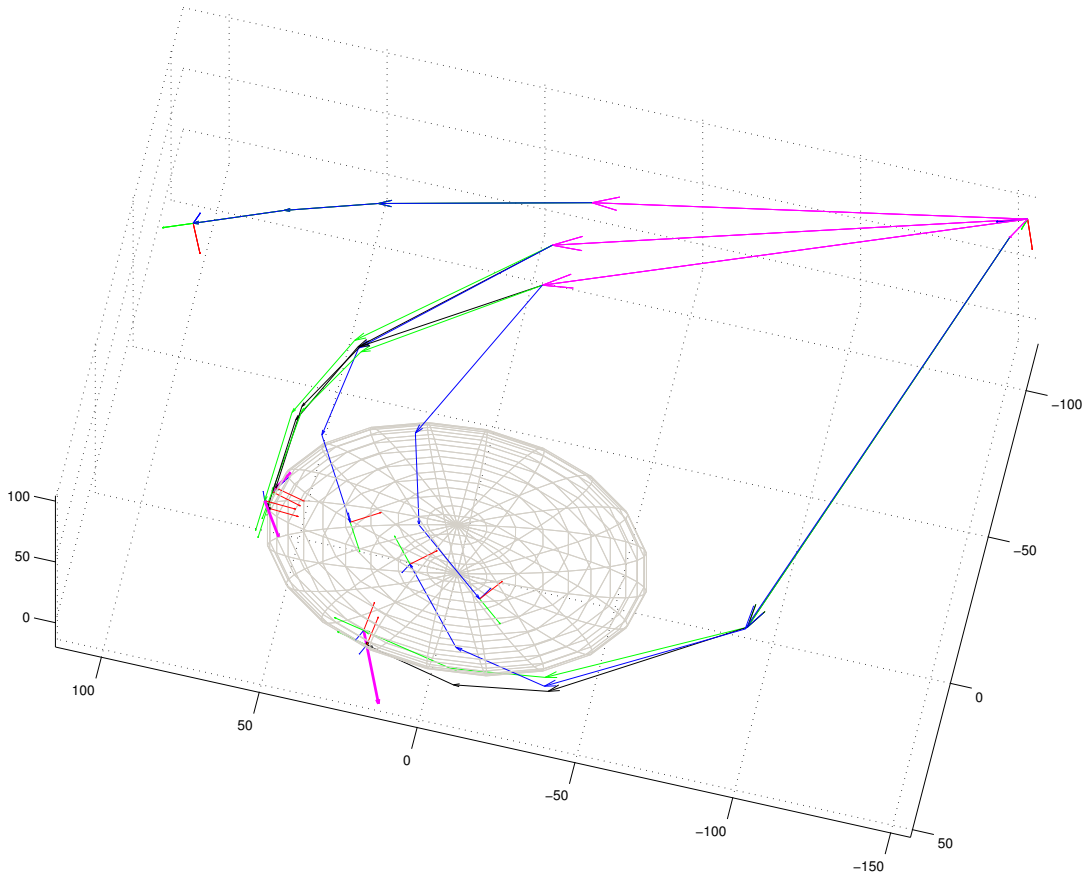

Fig. 5. A solution obtained for the Schunk Anthropomorphic hand satisfying all constraints performing the grasp with three fingers (thumb, index and middle). The color code corresponds to that of Fig. 2

dimension of the solution space in this case is $n_{s}=11$. The kinematic structure, spring constants and friction coefficients needed to write the grasp synthesis problem as stated in Section $\amalg$ are shown in Table In this case, the initial guess was set by introducing the constraints sequentially, such that it was as close as possible from the solution manifold. The result using the proposed method is shown in Fig. 5

\section{CONCLUSIONS AND FUTURE WORK}

The proposed approach tackles simultaneously the contact reachability, the object restrainment and the force controllability constraints that a feasible and prehensile grasp must satisfy. This is obtained by introducing torsional springs modeling the joint compliance, and spatial springs for the contact interaction. This leads to a solution where all vari- ables ultimately employs configuration values, and therefore, the hand can be commanded to grasp the object using only a position controller.

The results show practicable solutions provided by the proposed method for illustrative examples, suggesting how to: (i) reach the specified regions on object with the fingertips, (ii) apply the forces in the directions allowed by the contact model within the friction constraints, and (iii) compensate such forces using the hand joints, i.e. the hand performs a feasible and prehensile grasp of the object with the minimum effort.

A contact in the palm will affect significantly the approach, since it is the common element for all fingers. However, it would be worth studying such influence since this is the case for certain human grasps. 
TABLE III

PARAMETERS FOR EXAMPLE 2.

a) Kinematic structure and limit values

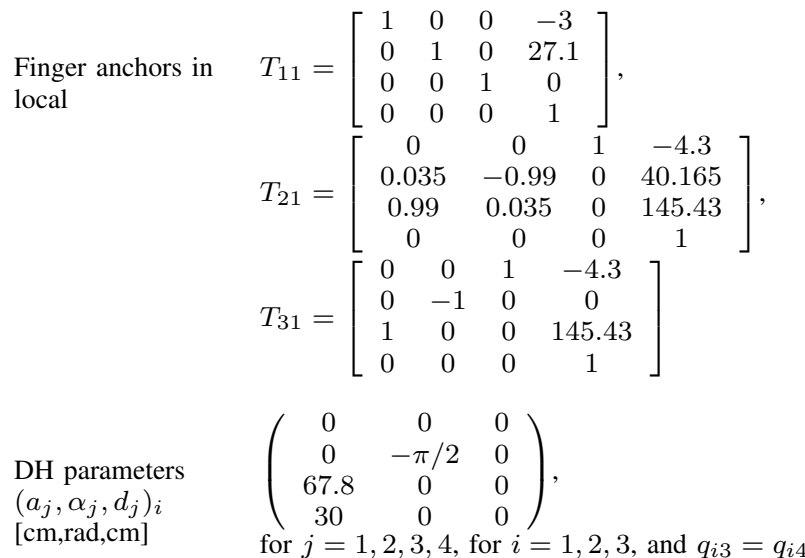

Joint limits [deg] $\mathbf{q}^{l}=\left[\begin{array}{c}0 \\ -15 \\ -4 \\ 4 \\ -15 \\ -4 \\ 4 \\ -15 \\ -4 \\ 4\end{array}\right], \mathbf{q}^{u}=\left[\begin{array}{c}90 \\ 15 \\ 75 \\ 75 \\ 15 \\ 75 \\ 75 \\ 15 \\ 75 \\ 75\end{array}\right]$

Torque limits

$[\mathrm{Ncm}]$

$\boldsymbol{\tau}^{\min }=10[\mathbf{1}], \boldsymbol{\tau}^{\max }=1000[\mathbf{1}]$,

where $[\mathbf{1}] \in \mathbb{R}^{m}$ is a vector containing ones

Contact point

$\mathbf{x}_{c}, \forall c$ in local

$[\mathrm{cm}]$

$\mathbf{x}=\left[\begin{array}{c}0 \\ 29.5 \\ 0\end{array}\right]$

Regions $S_{c}, \forall c$

$\mathbf{s}=\left[\begin{array}{c}60 \cos \left(\gamma_{1, c}\right) \sin \left(\gamma_{2, c}\right) \\ 40 \sin \left(\gamma_{1, c}\right) \sin \left(\gamma_{2, c}\right) \\ 20 \cos (\gamma 2, c)\end{array}\right]$,

with $\gamma_{1, c} \in[0,2 \pi]$ and $\gamma_{2, c} \in[0, \pi]$

\section{b) Coefficients}

Joint stiffness

[N/rad]

Friction (PCWF)

$\mu_{c}, \forall c$

Contact stiffness $\quad \tilde{\mathbf{K}}=\operatorname{blkdiag}(5,5,5)$

$\tilde{\mathbf{K}}_{c}, \forall c[\mathrm{~N} / \mathrm{cm}]$
[7] J. P. Saut, A. Sahbani, S. El-Khoury, and V. Perdereau, "Dexterous manipulation planning using probabilistic roadmaps in continuous grasp subspaces," in Proc. of the IEEE/RSJ Intl. Conf. on Int. Robots and Sys., 2007.

[8] H. R. Choi, W. K. Chung, and Y. Youm, "Stiffness analysis and control of multi-fingered robot hands," in Amer. Ctrl. Conf., 1994, vol. 1, 1994 pp. 621-626 vol.1.

[9] M. R. Cutkosky and I. Kao, "Computing and controlling compliance of a robotic hand," IEEE Trans. on Robotics and Aut., vol. 5, no. 2, pp. 151-165, 1989.

[10] V. Nguyen, "Constructing force- closure grasps," The Intl. J. of Robotics Res., vol. 7, no. 3, pp. 3-16, 1988.

[11] N. S. Pollard, "Closure and quality equivalence for efficient synthesis of grasps from examples," The Intl. J. of Robotics Res., vol. 23, no. 6, pp. 595-613, 2004.

[12] M. A. Roa and R. Suárez, "Computation of independent contact regions for grasping 3-D objects," IEEE Trans. on Robotics, vol. 25 no. 4, pp. 839-850, 2009.

[13] C. Borst, M. Fischer, and G. Hirzinger, "Calculating hand configurations for precision and pinch grasps," in Proc. of the IEEE/RSJ Intl. Conf. on Int. Robots and Sys., vol. 2, 2002, pp. 1553-1559.

[14] Y. Guan and H. Zhang, "Kinematic feasibility analysis of 3-D multifingered grasps," IEEE Trans. on Robotics and Aut., vol. 19, no. 3, pp. 507-513, 2003

[15] C. Rosales, L. Ros, J. M. Porta, and R. Suárez, "Synthesizing grasp configurations with specified contact regions," The Intl. J. of Robotics Res., vol. 30, no. 4, pp. 431-443, 2011.

[16] J. A. Claret and R. Suárez, "Efficient and practical determination of grasping configurations for anthropomorphic hands," in Proc. of the 18th IFAC World Congress, 2011.

[17] J. Kerr and B. Roth, "Analysis of multifingered hands," The Intl. J. of Robotics Res., vol. 4, no. 4, pp. 3-17, 1986.

[18] V. Kumar and K. J. Waldron, "Suboptimal algorithms for force distribution in multifingered grippers," IEEE Trans. on Robotics and Aut., vol. 5, no. 4, pp. 491-498, 1989.

[19] A. Bicchi, "On the problem of decomposing grasp and manipulation forces in multiple whole-limb manipulation," Robotics and Aut. Sys., vol. 13 , no. 2, pp. 127-147, 1994.

[20] M. Buss, H. Hashimoto, and J. B. Moore, "Dextrous hand grasping force optimization," IEEE Trans. on Robotics and Aut., vol. 12, no. 3 , pp. 406-418, 1996

[21] M. Gabiccini and A. Bicchi, "On the role of hand synergies in the optimal choice of grasping forces," in Proc. of Robotics: Science and Sys., 2010.

[22] E. Boivin, I. Sharf, and M. Doyon, "Optimum grasp of planar and revolute objects with gripper geometry constraints," in Proc. of the IEEE Intl. Conf. on Robotics and Aut., 2004, pp. 326-332 Vol.1.

[23] M. A. Roa, K. Hertkorn, C. Borst, and G. Hirzinger, "Reachable independent contact regions for precision grasps," in Proc. of the IEEE Intl. Conf. on Robotics and Aut., 2011, pp. 5337-5343.

[24] Y.-B. Y. Jia, F. Guo, and J. Tian, "On two-finger grasping of deformable planar objects," in Proc. of the IEEE Intl. Conf. on Robotics and Aut., 2011, pp. 5261-5266.

[25] M. Ciocarlie and P. Allen, "A constrained optimization framework for compliant underactuated grasping," Mech. Sciences, vol. 2, no. 1, pp. 17-26, 2011.

[26] S. H. Juan and J. M. Mirats-Tur, "A method to generate stable, collision free configurations for tensegrity based robots," in Proc. of the IEEE/RSJ Intl. Conf. on Int. Robots and Sys., 2008, pp. 3769-3774.

[27] A. Bicchi, M. Gabiccini, and M. Santello, "Modelling natural and artificial hands with synergies," Phil. Trans. of the Royal Society B: Bio. Sci., vol. 366, no. 1581, pp. 3153-3161, 2011.

[28] M. Santello, M. Flanders, and J. F. Soechting, "Postural hand synergies for tool use," J. of Neuroscience, vol. 18, no. 23, pp. 10105-10115, 1998.

[29] J. Rosell, R. Suárez, C. Rosales, and A. Pérez, "Autonomous motion planning of a hand-arm robotic system based on captured human-like hand postures," Aut. Robots, vol. 31, no. 1, pp. 87-102, 2011.

[30] M. R. Cutkosky, "On grasp choice, grasp models, and the design of hands for manufacturing tasks," IEEE Trans. on Robotics and Aut., vol. 5, no. 3, pp. 269-279, 1989.

[31] A. Bicchi, "On the closure properties of robotic grasping," The Intl. J. of Robotics Res., vol. 14, no. 4, pp. 319-334, 1995.

[32] J. Nocedal and S. J. Wright, Numerical Optimization, 2nd ed., ser. Springer Series in Oper. Res. Springer, 2006. 